\title{
Regional, sex, and age differences in diagnostic testing among participants in the NAVIGATE-ESUS trial
}

International Journal of Stroke 2021, Vol. 16(1) 55-62 (C) 2019 World Stroke Organization Article reuse guidelines: sagepub.com/journals-permissions DOI: $10.1177 / 1747493019884523$ journals.sagepub.com/home/wso @SAGE

\author{
Antonio Arauz' ${ }^{\oplus}$, Fabiola Serrano', Lesly A Pearce ${ }^{2}$, \\ Scott E Kasner ${ }^{3}$, Sebastian F Ameriso ${ }^{4}$, Danilo Toni ${ }^{5}$, \\ Daniel Bereczki ${ }^{6}$, James Siegler ${ }^{3}$, Angélica Ruiz-Franco ${ }^{7}$, \\ Carlos Cantú-Brito ${ }^{8}$, Anna Czlonkowska9, Wilfried Lang ${ }^{10}$, \\ Scott D Berkowitz ${ }^{11}$, Hardi Mundl ${ }^{12}$ and Robert G Hart ${ }^{13}$
}

\begin{abstract}
Background and aim: The diagnosis of embolic stroke of undetermined source (ESUS) is based on excluding other more likely stroke etiologies, and therefore diagnostic testing plays an especially crucial role. Our objective was to compare the diagnostic testing by region, sex, and age among the participants of NAVIGATE-ESUS trial.

Methods: Participants were grouped according to five global regions (North America, Latin America, Western Europe, Eastern Europe and East Asia), age $(<60,60-74$, and $>75$ years), and sex. Frequencies of each diagnostic test within areas of echocardiography, cardiac rhythm monitoring, and arterial imaging were described and compared across groups. A multivariable logistic regression model for each diagnostic test was fit to assess the independent influence of each of region, age, and sex and likelihood of testing.

Results: We included 6985 patients in the analysis (918 from North America; 746 from Latin America; 2853 from Western Europe; 1118 from Eastern Europe; 1350 from East Asia). Average age (highest in Western Europe (69 years), lowest in Eastern Europe (65 years)), \% females (highest in Latin America (44\%) and lowest in East Asia (3I\%)), and use of each diagnostic test varied significantly across regions. Region, but not sex, was independently associated with use of each diagnostic test examined. Transesophageal echocardiography and either CT or MR angiogram were more often used in younger patients.
\end{abstract}

Conclusion: Diagnostic testing differed by region, and less frequently by age, but not by sex. Our findings reflect the existing variations in global practice in diagnostic testing in ESUS patients.

\section{Keywords}

Embolic stroke of undetermined source, region, age, sex

Received: 16 May 2019; accepted: 27 August 2019

\section{Introduction}

The clinical construct of embolic stroke of undetermined source (ESUS) was proposed to define non-lacunar cryptogenic ischemic stroke likely due to embolism. Because the diagnosis is based on excluding other more likely stroke etiologies, ESUS comprises

\footnotetext{
'Stroke Clinic, Instituto Nacional de Neurología y Neurocirugía Manuel Velasco Suarez, México City, México

${ }^{2}$ Biostatistics Consultant, Minot, USA

${ }^{3}$ Department of Neurology, University of Pennsylvania, PA, USA

${ }^{4}$ Institute for Neurological Research FLENI, Buenos Aires, Argentina

${ }^{5}$ Department of Human Neurosciences, Sapienza University of Rome, Rome, Italy
}

\footnotetext{
${ }^{6}$ Department of Neurology, Semmelweis University, Budapest, Hungary ${ }^{7}$ Neurology Department, Hospital Juárez, México City, México

${ }^{8}$ Department of Neurology. Instituto Nacional de Ciencias Medicas y Nutrición Salvador Zubiran, Mexico City, Mexico

${ }^{9}$ Department of Neurology, Institute Psychiatry and Neurology, Warsaw, Poland

${ }^{10}$ Department of Neurology, Sigmund Freud University Vienna, Medical Faculty and Hospital St. John of God, Beaconsfield, Australia

"'Bayer U.S., LLC, Whippany, New Jersey, NJ, USA

${ }^{12}$ Bayer AG, Wuppertal, Germany

${ }^{13}$ Population Health Research Institute, David Braley Cardiac, Vascular and Stroke Research Institute, Hamilton, Canada

Corresponding author:

Antonio Arauz, Stroke Clinic, Instituto Nacional de Neurologia y Neurocirugia Manuel Velasco Suárez, Mexico City.

Email: antonio.arauz@prodigy.net.mx
} 
heterogeneous pathologies, and therefore diagnostic testing plays an especially crucial role. ${ }^{1}$

Screening for covert atrial fibrillation (AF) is a key step in the diagnosis of ESUS. The optimal techniques for ECG monitoring are uncertain given the lack of head-to-head trials and recent rapid advances in device design. Three major randomized controlled trials have examined the value of undertaking further monitoring in patients with cryptogenic stroke. The results show significantly higher detection rates with more intensive rhythm monitoring strategies than with standard care. ${ }^{2-4}$ In most regions, the first-line method for non-invasive detection of AF in ESUS patients remains via Holter monitoring. Non-invasive monitoring using loop recorders can record cardiac rhythm for several weeks, while other sites prefer longer term monitoring via implanted cardiac monitors.

Recent data suggest that the etiology of ESUS could be beyond cardioembolism, be heterogeneous and have other sources of embolism, or the result of nonstenosing large-artery atherosclerosis, aortic atherosclerosis, or nonatherosclerotic vasculopaties. ${ }^{5}$ This suggests that other studies such as transesophageal echocardiography (TEE) and intracranial vascular imaging could be of importance in the study of patients with ESUS. However, many of these diagnostic methods are not readily accessible in many low and middle-income countries. In the ESUS global registry, for up to $14 \%$ of the patients who did not meet ESUS criteria, the reason was insufficient diagnostic testing. ${ }^{6}$

Differences in the diagnostic testing in ESUS patients may vary not only from region to region, but also with other factors such as age and sex. Therefore, we compared the diagnostic testing according to region, sex, and age among the participants of NAVIGATEESUS trial.

\section{Methods}

The methods of the NAVIGATE ESUS trial and baseline characteristics of its participants have been published. $^{7}$ The trial enrolled 7213 patients between December 2014 and September 2017 who experienced a recent ischemic stroke and satisfied the criteria for ESUS, specifically, a nonlacunar stroke on brain imaging, open arteries proximal to the infarct, and no major-risk cardioembolic source. In addition to brain computed tomography or magnetic resonance imaging showing the brain infarct, patients required imaging of intracranial and extracranial cerebral arteries, to exclude extracranial vessel atherosclerosis causing more than $50 \%$ luminal stenosis in arteries supplying the area of ischemia. Intracranial imaging was otherwise optional, but if performed, a finding of more than $50 \%$ stenosis due to atherosclerosis was an exclusion criterion. An electrocardiogram, and at least 20 total hours of cardiac rhythm monitoring were also required in order to rule out AF lasting 6 min or longer, although investigators could choose to monitor for longer periods. Patients with implanted cardiac rhythm monitors were excluded from NAVIGATE ESUS study. No participant had an implantable cardiac rhythm monitor. Non-implantable monitors were used for 7,14 , or 30 days, and the patient could not be randomized until cardiac rhythm monitoring was completed. Echocardiography was required, either transthoracic (TTE) or TEE.

Data were analyzed according to five global regions (Figure 1): North America (Canada and United States of America (USA)), Latin America (Argentina, Brazil, Chile, México), Western Europe (Austria, Belgium, Denmark, Finland, France, Germany, Greece, Ireland, Italy, Portugal, Spain, Sweden, Switzerland, United Kingdom), Eastern Europe (Czech Republic, Hungary, Poland, Russian Federation, Turkey), and East Asia (China, Japan, South Korea). Participants from Australia $(n=119)$, Israel $(n=60)$, and South Africa $(n=49)$ could not be categorized into one of these five geographical regions and were excluded from these analyses. Descriptive statistics summarizing age (mean \pm standard deviation (SD)), male sex, and frequency of each diagnostic test were done overall and by each of region, sex, and age (categorized a priori as $<60,60-74$, and $>75$ years), and compared across groups using an ANOVA or Chi-square test as appropriate. A multivariable logistic regression model for each diagnostic test was fit to assess the influence of region (4 degrees of freedom, df), age ( $2 \mathrm{df}$ ), and sex (1 df) on likelihood of that test with Western Europe (largest region), age $<60$ years (youngest), and male sex serving as reference groups. Results from the models were summarized qualitatively by considering which groups were significantly different from the reference groups. Statistical significance was accepted at the 0.01 level (two-sided), and no adjustment was made for multiple comparisons. Analyses were done using SPSS 24.0.0.0 software.

\section{Results}

This analysis included $6985(97 \%)$ of the 7213 NAVIGATE-ESUS clinical trial participants: 918 from North America; 746 from Latin America; 2853 from Western Europe; 1118 from Eastern Europe; 1350 from East Asia. Age (mean $+\mathrm{SD}$ ) varied across region $(p<0.001)$ with the highest in Western Europe $(69+10$ years $)$ and lowest in Eastern Europe $(65+8$ years). The fraction of participants who were male also varied $(p<0.001)$ and was highest in East Asia $(71 \%)$ and lowest in Latin America (56\%). (Table 1) 
Figure I. Number of analyzed patients by region.

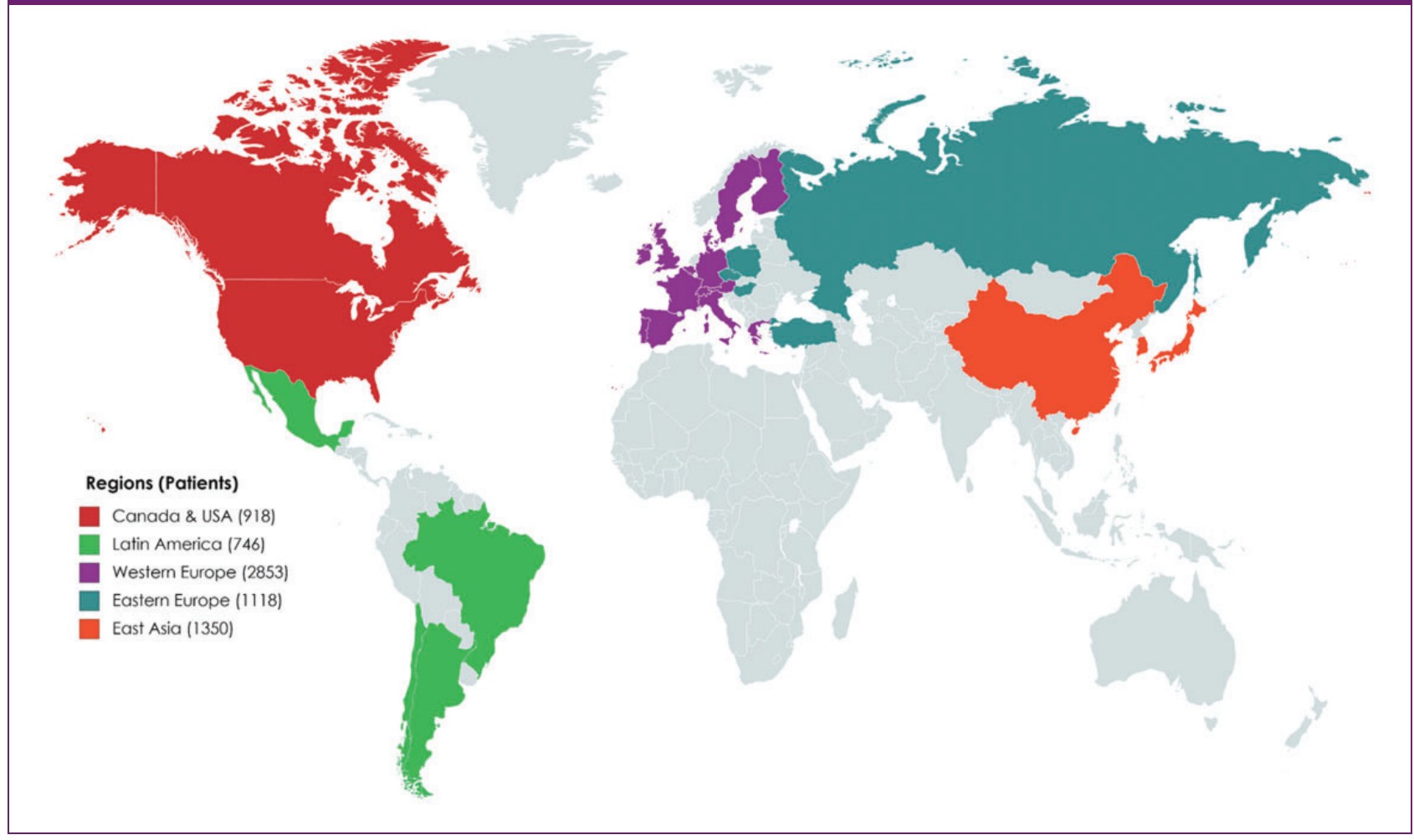

\section{Diagnostic testing by region}

The relative frequencies of all diagnostic tests varied by region (Table 1). TTE was common across all regions (range 93-99\%), while TEE was nearly twice as frequent in East Asia (26\%) and Western Europe (23\%) as in Eastern Europe (12\%) or Latin America (13\%). Participants in East Asia were more likely to have both TTE and TEE $(25 \%)$.

For cardiac rhythm monitoring, in-hospital telemetry was most frequent in Western Europe $(53 \%)$ and infrequently used in Latin America $(7 \%)$, whereas ambulatory ECG (Holter) was most common in East Asia (98\%), Latin America (96\%) and Eastern Europe $(93 \%)$ where in-hospital telemetry was less common (range 7 to $13 \%$ ). Prolonged cardiac rhythm monitoring was frequently done in Canada/USA $(39 \%$ of participants) but uncommon elsewhere (range $<1$ to $8 \%$ ). Arterial imaging via CT angiogram was frequent in Canada/USA $(75 \%)$ but much less frequent elsewhere (range 23 to $38 \%$ ), and was frequent via MRI angiogram in East Asia (62\%) but about half as common elsewhere (range 10 to $36 \%$ ). CT or MR angiography was done in $89 \%$ of participants from Canada/USA versus $33 \%$ of those from Eastern Europe. Sonography was widely used in Eastern Europe $(82 \%)$, East Asia (79\%), Western Europe (68\%), and
Latin America (62\%), but infrequently used in Canada/ USA $(19 \%)$.

\section{Diagnostic testing by age and sex}

Fewer differences in frequencies of diagnostic tests were apparent considering patient age and sex (Table 2). Notably, TEE was done more frequently in patients under age 60 years $(24 \%)$ vs. those age 75 years or older $(14 \%)$. Both studies (TTE and TEE) were performed in a fewer patients, although more frequently in those under 60 years of age $(18 \%)$ vs. those age 75 years or older $(11 \%)$. Other absolute differences in frequencies were smaller.

\section{Multivariable analysis of the influence of region, age, and sex}

Region was independently associated (each $p<0.001$, $4 \mathrm{df}$ ) with likelihood of each of the diagnostic tests and combinations considered, whereas sex was not independently associated (each $p>0.18,1 \mathrm{df}$ ) with any of them. Age was independently associated with each of the echocardiography tests and the combination (each $p<0.001,2 \mathrm{df}$ ), but not any of the rhythm monitoring tests (each $p>0.19$ ). Age was also associated with likelihood of CT angiogram $(p<0.001,2 \mathrm{df})$ and CT 
Table I. Frequency of diagnostic testing by global region

\begin{tabular}{|c|c|c|c|c|c|c|c|}
\hline & $\begin{array}{l}\text { Overall } \\
(n=6985)\end{array}$ & $\begin{array}{l}\text { North } \\
\text { America } \\
(n=9 \mid 8)\end{array}$ & $\begin{array}{l}\text { Latin } \\
\text { America } \\
(n=746)\end{array}$ & $\begin{array}{l}\text { Western } \\
\text { Europe } \\
(n=2853)\end{array}$ & $\begin{array}{l}\text { Eastern } \\
\text { Europe } \\
(n=1 \mid 18)\end{array}$ & $\begin{array}{l}\text { East } \\
\text { Asia } \\
(n=1350)\end{array}$ & $p$-value* \\
\hline Age, mean $\pm S D$ & $67 \pm 10$ & $66 \pm 10$ & $66 \pm 10$ & $69 \pm 10$ & $65 \pm 8$ & $66 \pm 10$ & $<0.001$ \\
\hline Male, \% & 61 & 58 & 56 & 60 & 61 & 69 & $<0.001$ \\
\hline \multicolumn{8}{|l|}{ Echocardiography } \\
\hline Transthoracic, \% & 95 & 97 & 93 & 94 & 94 & 99 & $<0.001$ \\
\hline Transesophageal, \% & 20 & 15 & 13 & 23 & 12 & 26 & $<0.001$ \\
\hline Both, \% & 15 & 12 & 6 & 17 & 6 & 25 & $<0.00$ I \\
\hline \multicolumn{8}{|l|}{ Cardiac Rhythm Monitoring } \\
\hline In-hospital telemetry, \% & 31 & 34 & 7 & 53 & 12 & 13 & $<0.001$ \\
\hline \multicolumn{8}{|l|}{ If yes, duration, \% } \\
\hline$<24 \mathrm{hr}$ & 7 & 5 & 18 & 5 & 31 & 7 & \\
\hline $24-<48 \mathrm{hr}$ & 36 & 34 & 41 & 38 & 52 & 17 & \\
\hline$\geq 48 \mathrm{hr}$ & 54 & 55 & 41 & 55 & 16 & 72 & \\
\hline Uncertain/unknown & 3 & 7 & 0 & 2 & 1 & 3 & \\
\hline Ambulatory ECG (Holter), \% & 79 & 64 & 96 & 64 & 93 & 98 & $<0.001$ \\
\hline Duration, hours, median (IQR) & $24(I)$ & $24(24)$ & $24(1)$ & $24(1)$ & $24(1)$ & $24(I)$ & \\
\hline Other prolonged (>24hr), \% & 9 & 39 & $<1$ & 8 & 1 & 1 & $<0.001$ \\
\hline Duration, days, median (IQR) & $16(23)$ & $30(16)$ & & $7(8)$ & $7(9)$ & $7(4)$ & \\
\hline \multicolumn{8}{|l|}{ Arterial imaging } \\
\hline CT angiogram, \% & 37 & 75 & 34 & 38 & 24 & 23 & $<0.001$ \\
\hline MR angiogram, \% & 34 & 20 & 24 & 36 & 10 & 62 & $<0.001$ \\
\hline $\mathrm{CT}$ and/or MR angiogram, \% & 64 & 89 & 56 & 65 & 33 & 77 & $<0.001$ \\
\hline Sonography, \% & 64 & 19 & 62 & 68 & 82 & 71 & $<0.001$ \\
\hline
\end{tabular}

*Statistical significance for comparison across regions.

CT: computed tomography; MR: magnetic resonance; IQR: interquartile range; hr: hour; ECG: electrocardiography.

and/or MR angiogram $(p<0.001,2 \mathrm{df})$, but not with MR angiogram only $(p=0.53,2 \mathrm{df})$. Table 3 summarizes these differences qualitatively with exact results reported in the Online Supplemental Appendix.

\section{Discussion}

NAVIGATE-ESUS was a randomized clinical trial that compared different antithrombotic treatments in patients meeting the diagnostic criteria for ESUS. ${ }^{8}$
The study included a large cohort of patients across multiple continents. Despite the fact that minimum diagnostic studies were required for its inclusion, the trial cohort provides ample opportunity to assess the differences in diagnostic testing in four continents.

Prolonged cardiac rhythm monitoring is infrequently used in all regions. In Latin America, Eastern Europe, and East Asia it was reported for less than 1\% of patients, while in Canada/USA, where it was most frequently done, the percentage reached $39 \%$. 
Table 2. Frequency of diagnostic testing by age and sex

\begin{tabular}{|c|c|c|c|c|c|c|c|c|}
\hline & \multirow[b]{2}{*}{$\begin{array}{l}\text { Overall } \\
(n=6985)\end{array}$} & \multicolumn{4}{|c|}{ Age categories } & \multicolumn{3}{|l|}{ Sex } \\
\hline & & $\begin{array}{l}<60 \text { years } \\
(n=1660)\end{array}$ & $\begin{array}{l}60-74 \\
\text { years } \\
(n=3679)\end{array}$ & $\begin{array}{l}\geq 75 \text { years } \\
(n=1646)\end{array}$ & $p$-value* & $\begin{array}{l}\text { Male } \\
(n=4290)\end{array}$ & $\begin{array}{l}\text { Female } \\
(n=2695)\end{array}$ & $p$-value ${ }^{\#}$ \\
\hline \multicolumn{9}{|l|}{ Echocardiography } \\
\hline Transthoracic, \% & 95 & 94 & 95 & 97 & $<0.001$ & 96 & 95 & 0.5 \\
\hline Transesophageal, \% & 20 & 24 & 20 & 14 & $<0.001$ & 20 & 18 & 0.01 \\
\hline Both, \% & 15 & 18 & 15 & 11 & $<0.001$ & 16 & 13 & 0.001 \\
\hline \multicolumn{9}{|l|}{ Cardiac rhythm monitoring } \\
\hline In-hospital telemetry, \% & 31 & 28 & 31 & 35 & $<0.001$ & 31 & 33 & 0.1 \\
\hline \multicolumn{9}{|l|}{ If yes, duration, \% } \\
\hline$<24 \mathrm{hr}$ & 7 & 8 & 8 & 5 & & 8 & 7 & \\
\hline $24-<48 \mathrm{hr}$ & 36 & 33 & 39 & 34 & & 38 & 34 & \\
\hline$\geq 48 \mathrm{hr}$ & 54 & 56 & 50 & 59 & & 52 & 57 & \\
\hline Uncertain/unknown & 3 & 3 & 3 & 2 & & 3 & 2 & \\
\hline Ambulatory ECG (Holter), \% & 79 & 81 & 79 & 76 & 0.003 & 79 & 78 & 0.3 \\
\hline Duration, hours, median (IQR) & $24(I)$ & $24(I)$ & $24(1)$ & $24(1)$ & & $24(I)$ & $24(1)$ & \\
\hline Other prolonged (>24hr), \% & 9 & 9 & 9 & 9 & 0.9 & 9 & 9 & 0.6 \\
\hline Duration, days, median (IQR) & $16(23)$ & $22(23)$ & $19(23)$ & $14(26)$ & & $14(23)$ & $21(23)$ & \\
\hline \multicolumn{9}{|l|}{ Arterial imaging } \\
\hline $\mathrm{CT}$ angiogram, \% & 37 & 40 & 38 & 33 & $<0.001$ & 37 & 38 & 0.3 \\
\hline MR angiogram, \% & 34 & 33 & 33 & 35 & 0.5 & 35 & 32 & 0.02 \\
\hline $\mathrm{CT}$ and/or MR angiogram, \% & 64 & 65 & 65 & 62 & 0.05 & 65 & 64 & 0.5 \\
\hline Sonography, \% & 64 & 64 & 63 & 65 & 0.2 & 64 & 63 & 0.2 \\
\hline
\end{tabular}

*Statistical significance for comparison across age categories.

\#Statistical significance for comparison between male and females.

CT: computed tomography; MR: magnetic resonance; IQR: interquartile range; hr: hour; ECG: electrocardiography.

Subclinical AF is the leading occult mechanism for ESUS. Multiple studies show that prolonged heartrhythm monitoring establishes a new diagnosis of AF in $10 \%$ to $20 \%$ of patients with recent stroke. ${ }^{4,9}$ The yield increases with the duration of monitoring and approaches 30\% after three years of continuous monitoring. ${ }^{2,10}$ In our study, the difference in the other types of cardiac rhythm monitoring was also evident: in Latin America, in-hospital telemetry was done in only $7 \%$ of the patients, whereas ambulatory ECG Holter (with median duration of $24 \mathrm{~h}$ ) was widely done in Latin America, Eastern Europe, and East Asia. These differences can be explained by the access to the different diagnostic methods in the different regions. In low- and middle-income countries, the prolonged monitoring via implanted cardiac monitor is infrequently used and underdiagnosed AF is likely to contribute significantly to cryptogenic stroke and ESUS. ${ }^{11}$

Diagnostic testing may affect the trial outcome as patients with more extensive investigation probably represent a slightly different ESUS cohort. The value of TEE in patients with ESUS was previously evaluated in a small cohort. ${ }^{12}$ Abnormal TEE findings may decisively affect the selection of appropriate therapeutic strategy in one of seven patients with ESUS. Given that the diagnostic criteria of ESUS allows TTE, 
Table 3. Qualitative summary of factors independently associated with undergoing diagnostic testing after multivariate analysis. ${ }^{a}$

\begin{tabular}{|c|c|c|c|}
\hline & Age & Sex & Region \\
\hline \multicolumn{4}{|l|}{ Echocardiography } \\
\hline Transthoracic & $\uparrow$ with older age & No & $\uparrow$ in East Asia and USA and Canada \\
\hline Transesophageal & $\downarrow$ with older age & No & $\uparrow$ in East Asia and Western Europe \\
\hline Both & $\downarrow$ with older age & No & $\uparrow$ in East Asia and Western Europe \\
\hline \multicolumn{4}{|l|}{ Cardiac rhythm monitoring } \\
\hline In-hospital telemetry & No & No & $\uparrow$ in Western Europe \\
\hline Ambulatory ECG (Holter) & No & No & $\uparrow$ in East Asia, Latin America, and Eastern Europe \\
\hline Other prolonged (>24hr) & No & No & $\uparrow \uparrow$ in USA and Canada; $\uparrow$ in Western Europe \\
\hline \multicolumn{4}{|l|}{ Arterial imaging } \\
\hline $\mathrm{CT}$ angiogram & $\downarrow$ with age $\geq 75$ & No & $\uparrow \uparrow$ in USA and Canada; $\uparrow$ Western Europe \\
\hline MR angiogram & No & No & $\uparrow \uparrow$ in East Asia; $\uparrow$ Western Europe \\
\hline $\mathrm{CT}$ and/or MR angiogram & $\downarrow$ with age $\geq 75$ & No & $\uparrow \uparrow$ in USA and Canada; $\uparrow$ East Asia and Western Europe \\
\hline Sonography & No & No & $\uparrow \uparrow$ in Eastern Europe; $\uparrow$ East Asia and Western Europe \\
\hline
\end{tabular}

CT: computed tomography; MR: magnetic resonance; ECG: electrocardiography; hr: hour.

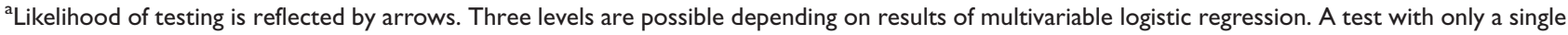
arrow for a region indicates an increased odds for the listed region(s) relative to the other regions not listed, i.e. high $\uparrow$ and reference (not listed). A test with both double and single arrows indicates three groups, i.e. highest odds $\uparrow \uparrow$, higher $\uparrow$, and reference (not listed). See Supplemental Appendix for the specific numbers.

the TEE was only used in $20 \%$ of NAVIGATE-ESUS patients, and in Latin America and Eastern Europe was done in only $13 \%$ and $12 \%$, respectively. Extensive evaluation including TEE and cardiac monitoring for longer periods may help to identify the etiology of the stroke in these patients. Using routine TTE, it is possible to identify only a small subset of patients with ESUS without known AF who might benefit from treatment with anticoagulants. A secondary analysis of NAVIGATE-ESUS ${ }^{13}$ found that in patients with at least moderate left atrial enlargement, there was a significant reduction in ischemic stroke with rivaroxaban. These results suggest that left atrial volume would have been a better marker of left atrial size; however, this was not available for a large number of ESUS patients.

The results of NAVIGATE-ESUS and RESPECTESUS $^{14}$ suggest that ESUS is not a single entity that can be approached with a single antithrombotic treatment strategy. Recent studies indicate that some ESUS cases result from subclinical cardioembolism (atrial cardiomyopathy, unrecognized myocardial infarction, patent foramen ovale), while other cases result from nonstenosing large-artery atherosclerosis (aortic atherosclerosis or nonatherosclerotic vasculopathies). ${ }^{14}$
In this sense, some extension of diagnostic studies could be useful, especially arterial imaging. Recently, Singh et al. reported that among patients with ESUS, one in five had carotid intraplaque hemorrhage identified by MRI of the vessel wall ipsilateral to the ischemic stroke. This observation raises the possibility that mildly stenotic atherosclerotic plaque may be the stroke etiology in many ESUS patients. ${ }^{15}$ Our results show $64 \%$ of the patients had CT and/or MR angiogram; however, in Eastern Europe, this diagnostic testing was done in only $33 \%$ of the cases, versus $89 \%$ and $77 \%$ in North America and East Asia, respectively.

Although the disparities in the stroke quality of care between women and men have been well documented, ${ }^{16}$ we did not find an influence of the patient's sex on diagnostic testing independent of region. This result is encouraging but should be taken with caution, since it is a clinical trial that requires a minimum protocol of diagnostic tests as an inclusion criteria and may not reflect the daily practice of the countries participating in the trial.

We found preference for some diagnostic tests such a TEE and CT/MR angiogram in younger patients. Based on age, it is likely that younger patients with ESUS may have different causes (e.g. patent foramen ovale), 
which is better characterized with TEE. There are important differences between younger versus older patients with ESUS regarding risk factors and potential causes. Alternatively, younger stroke patients may more often undergo a more complete evaluation. The ongoing young ESUS registry could provide the global frequency and characteristics of younger patients with ESUS. ${ }^{17}$

Our results reflect the existing variation in global practice in diagnostic testing at major stroke research centers. In many centers, many stroke patients were not considered to be ESUS due to lack of diagnostic testing to meet ESUS criteria. The lack of diagnostic studies and the variation in their use are likely to be different in daily practice, mainly in low- and middle-income countries, where prolonged monitoring of heart rate and echocardiography is not routinely used for patients outside of clinical trials. In a Mexican registry of 1246 patients with cerebral infarction, etiology was unknown in $41 \%$ due to the lack of appropriate studies. ${ }^{18}$

Our study has potential limitations. This clinical trial cohort represents patients who are willing and able to participate in a clinical trial, which may limit the generalizability of our findings. A single site in each country also may not accurately reflect the practice in the country or the region. However, the study sites represented many global regions. In addition, NAVIGATEESUS required a minimum of diagnostic studies for its inclusion.

Characterizing patients with ESUS and developing therapies for specific mechanisms, can only be achieved with adequate and complete diagnostic testing. Our results highlight the variability of diagnostic testing. Some studies ${ }^{19}$ suggest a benefit of antiplatelet therapy in a subgroup of patients with ESUS, perhaps due to different predominant mechanism of stroke.

Although the ESUS definition alone cannot serve as a therapeutic indication for anticoagulation, it may provide a useful basis for further diagnostic advances that in turn could allow for more effective and personalized treatment for stroke prevention. Future trials will need to investigate various antiplatelet drugs, including the combination therapies of antiplatelet agents or antiplatelet therapies combined with low dose of anticoagulants. A recent secondary analysis of the Cardiovascular OutComes for People using Anticoagulation StrategieS (COMPASS) trial found that among patients with systemic atherosclerosis, low-dose rivaroxaban-plus-aspirin was associated with significant reductions in ESUS. ${ }^{20}$

In view of the fact that two large, recent randomized trials failed to demonstrate the superiority of anticoagulants over aspirin in patients with ESUS, our findings support a strategy of extending the diagnosis evaluation of patients with ESUS, in an attempt to more precisely categorize the cause of the stroke and to individualize secondary prevention.
The identification of ESUS patients at high risk for stroke recurrence is a challenge. In a recently proposed tool, age, leukoaraiosis, and multiterritorial infarct were identified as independent predictors. The tool allows classifying patients with low risk (2.1 per 100 patients-years), intermediate risk (3.74 per patientyears), and high risk (8.23 per 100 patients-years). ${ }^{21}$

\section{Conclusion}

We found that relative frequencies of all diagnostic tests varied by region, and less frequently age, but not by sex. Our results reflect the existing variations in global practice of diagnostic testing in ESUS patients that likely extend to clinical practice as well. Our findings support that diagnostic evaluation should be expanded in patients with ESUS, trying to more accurately categorize the cause of stroke in order to individualize secondary prevention.

\section{Declaration of conflicting interests}

The author(s) declared no potential conflicts of interest with respect to the research, authorship, and/or publication of this article.

\section{Funding}

The author(s) received no financial support for the research, authorship, and/or publication of this article.

\section{ORCID iD}

Antonio Arauz (D) https://orcid.org/0000-0002-3340-4138

\section{Supplemental material}

Supplemental material for this article is available online.

\section{References}

1. Hart RG, Diener H-C, Coutts SB, et al. Embolic strokes of undetermined source: the case for a new clinical construct. Lancet Neurol 2014; 13: 429-438.

2. Gladstone DJ, Spring M, Dorian P, et al. Atrial fibrillation in patients with cryptogenic stroke. $N$ Engl J Med 2014; 370: 2467-2477.

3. Wachter R, Gröschel K, Gelbrich G, et al. Holterelectrocardiogram-monitoring in patients with acute ischaemic stroke (Find-AF RANDOMISED): an openlabel randomised controlled trial. Lancet Neurol 2017; 16: 282-90.

4. Sanna T, Diener H-C, Passman RS, et al. Cryptogenic stroke and underlying atrial fibrillation. $N$ Engl J Med 2014; 370: 2478-2486.

5. Kamel H, Merkler AE, Iadecola C, Gupta A and Navi BB. Tailoring the approach to embolic stroke of undetermined source. JAMA Neurol 2019; 76: 855-861.

6. Perera KS, Vanassche T, Bosch J, et al. Embolic strokes of undetermined source: prevalence and patient features in the ESUS Global Registry. Int J Stroke 2016; 11: 526-533. 
7. Kasner SE, Lavados $\mathrm{P}$, Sharma $\mathrm{M}$, et al. Characterization of patients with embolic strokes of undetermined source in the NAVIGATE ESUS randomized trial. J Stroke Cerebrovasc Dis 2018; 27: 1673-1682.

8. Hart RG, Sharma M, Mundl H, et al. Rivaroxaban for stroke prevention after embolic stroke of undetermined source. N Engl J Med 2018; 378: 2191-2201.

9. Sposato LA, Cipriano LE, Saposnik G, Vargas ER, Riccio PM and Hachinski V. Diagnosis of atrial fibrillation after stroke and transient ischaemic attack: a systematic review and meta-analysis. Lancet Neurol 2015; 14 : 377-387.

10. Witsch J, Merkler AE, Chen ML, et al. Incidence of atrial fibrillation in patients with recent ischemic stroke versus matched controls. Stroke 2018; 49: 2529-2531.

11. Cantú-Brito C, Sampaio Silva G and Ameriso SF. Embolic stroke of undetermined source in Latin America. Neurologist 2017; 22: 171-81.

12. Katsanos AH, Bhole R, Frogoudaki A, et al. The value of transesophageal echocardiography for embolic strokes of undetermined source. Neurology 2016; 87: 988-995.

13. Healey JS, Gladstone DJ, Swaminathan B, et al. Recurrent stroke with rivaroxaban compared with aspirin according to predictors of atrial fibrillation. JAMA Neurol 2019; 76: 764-773.

14. Diener H-C, Sacco RL, Easton J, et al. Dabigatran for prevention of stroke after embolic stroke of undetermined source. N Engl J Med 2019; 380: 1906-1917.
15. Singh N, Moddy AR, Panzov V and Gladstone D. Carotid intraplaque hemorrhage in patients with embolic stroke of undetermined source. J Stroke Cerebrovasc Dis 2018; 27: 1956-1959.

16. McDermott M, Lisabeth LD, Baek J, et al. Sex disparity in stroke quality of care in a community-based study. J Stroke Cerebrovasc Dis 2017; 26: 1781-1786.

17. Perera KS, Swaminathan B, Veltkamp R, et al. Frequency and features of embolic stroke of undetermined source in young adults. Eur Stroke J 2018; 3: $110-116$.

18. Cantú-Brito C, Ruiz-Sandoval JL, Murillo-Bonilla LM, et al. The first Mexican multicenter register on ischaemic stroke (The PREMIER Study): demographics, risk factors and outcome. Int J Stroke 2011; 6: 93-94.

19. Rothwell PM, Algra A, Chen Z, et al. Effects of aspirin on risk and severity of early recurrent stroke after transient ischaemic attack and ischaemic stroke: time-course analysis of randomised trials. Lancet 2016; 388: 365-375.

20. Perera KS, Ng KKH, Nayar S, et al. Association between low-dose Rivaroxaban with or without aspirin and ischemic stroke subtypes: A Secondary Analysis of the COMPASS Trial. JAMA Neurol 2019 Sep 16. DOI: 10.1001/jamaneurol.2019.2984.

21. Ntaios G, Georgiopoulos G, Perlepe K, et al. A tool to identify patients with embolic stroke of undetermined source at high recurrence risk. Neurology 2019 (in press). 